\title{
Addendum to "On the Transition from Potential Flow to Turbulence ..." by M. Niemetz, R. Hänninen, and W. Schoepe, J. Low Temp. Phys. 187, 195 (2017)
}

\author{
W. Schoepe ${ }^{1}$ (I)
}

Received: 7 January 2018 / Accepted: 28 February 2018 / Published online: 7 March 2018

(C) Springer Science+Business Media, LLC, part of Springer Nature 2018

It should be mentioned that an interesting conclusion can be drawn from a comparison of the normalized mean lifetimes of the turbulent phases $\tau^{*}$ in Eq. (27)

$$
\tau^{*}=\exp \left(n^{2}\right)
$$

where $n$ is the number of vortex rings that are shed from the oscillating sphere during one half-period, and in Eq. (30):

$$
\tau^{*}=\exp \left[\left(c R e_{\mathrm{s}}\right)^{2}\right],
$$

where $c=1.04$ and $R e_{\mathrm{s}}$ is the superfluid Reynolds number. The accuracy of $\mathrm{c}$ is determined by the accuracy of several numerical factors in Eq. (26) and is estimated to be about $10 \%$. That means within our accuracy we may as well set $c=1$. Hence, the conclusion is:

$$
R e_{\mathrm{S}}=n .
$$

That means, in our experiment the superfluid Reynolds number is given simply by the number of vortex rings that are shed from the sphere in one half-period of oscillation. This is a surprisingly simple result. In a much different context, an equally simple result for $R e_{\mathrm{S}}$ has been obtained theoretically in 2D superfluid turbulence in a recent work by Reeves et al. Phys. Rev. Lett. 119, 184502 (2017).

$凶$ W. Schoepe

wilfried.schoepe@ur.de

1 Fakultät für Physik, Universität Regensburg, Regensburg, Germany 